\title{
PERFORMANCE DE POPULAÇÕES DE MILHO (Zea mays L.) EM TERRAS ALTAS SOB CERRADO NO CENTRO-SUL DO ESTADO DO TOCANTINS, BRASIL ${ }^{1}$
}

\author{
Camilo de L. MORELLO², Joênes M. PELUZIO ${ }^{3}$, Regina M.S. COÊLHO \\ Manoel X. dos SANTOS ${ }^{4}$
}

\begin{abstract}
RESUMO - No estado do Tocantins, o cultivo de milho em "terras altas sob cerrado" apresenta níveis de produtividade variáveis, cuja média não ultrapassa 2 t/ha. Fatores de solo, como acidez, baixos teores de $\mathrm{Ca}, \mathrm{Mg}, \mathrm{P}$ e $\mathrm{K}$ e matéria orgânica, concorrem para reduzir as produtividades. $\mathrm{O}$ desenvolvimento de materiais genéticos melhorados busca contribuir na superação dessas limitações. Como atividade inicial em um programa de melhoramento genético, o presente estudo avaliou um total de 160 populações de milho, nas safras 1994/95, 1995/96 e 1998/99, nos municípios de Pedro Afonso e Gurupi, em termos de produtividade de grãos (PG), altura da planta (AP), altura da espiga (AE) e dias para florescimento feminino (FF). Para PG, os efeitos de populações foram significativos em todas as avaliações, ocorrendo produtividades entre 1,59 t/ ha a 7,46 t/ha. Destacaram-se as populações IAPAR 51, EGO 17, CMS 08, CMS 50, COMPOSTO TERRAS BAIXAS, TAIÚBA, TAITINGA, CMS 28, CMS 15 e SIKALQ, em Pedro Afonso, e K9201, CMS 39, BR 105 e CMS 03, em Gurupi, com produtividades acima de 5 t/ha. As diferenças nas performances produtivas e agronômicas em Pedro Afonso e Gurupi, juntamente com efeitos significativos das interações populações x locais, alertam para a condução de programas de melhoramento específicos para cada local.
\end{abstract}

Palavras-chave: Milho, germoplasma, melhoramento, solos ácidos.

Performance of Maize Ppopulations (Zea mays L.) on Uplands under "Cerrado" in the Center-South of Tocantins State, Brazil

\begin{abstract}
In the state of Tocantins, the crop of maize in the high land under "cerrado" has levels of yield variable, whose average does not exceed $2 \mathrm{t} / \mathrm{ha}$. Events of soil, as acidity, low levels of $\mathrm{Ca}, \mathrm{Mg}, \mathrm{P}$ and $\mathrm{K}$ and organic matter, concur to decrease the yields. The development of genetics materials improved fetching to contribute in the overcoming of there limitations. As initial ativity in a program of genetic improvement, the present study it evaluated a whole of 160 maize populations, in seasons 1994/95, 1995/96 and 1998/99, in the cities of Pedro Afonso and Gurupi, in terms of grain weight $(\mathrm{GW})$, plant height $(\mathrm{PH})$, ear height $(\mathrm{EH})$ and days for feminine flowering (FF). For GW, the effects of the populations were significant in whole the evaluations, occurring yields between 1,59 t/ha the 7,46 t/ha. Populations IAPAR 51, EGO 17, CMS 08, CMS 50, COMPOSTO TERRAS BAIXAS, TAIÚBA, TAITINGA, CMS 28, CMS 15, and SIKALQ, had been distinguished in Pedro Afonso; and K 9201, CMS 39, BR 105, and CMS 03, in Gurupi, with yields above of the $5 \mathrm{t} / \mathrm{ha}$. The differences in the productive and agronomics performances in Pedro Afonso and Gurupi, together with significant effect of the interactions populations $x$ locals, alert for the conduction of the specifics improvement programs for each local.
\end{abstract}

Key-words: Maize, germoplasm, improvement, acid soil.

'Pesquisa executada com recursos do CNPq, projeto: Avaliação de Populações de Milho no Estado do Tocantins (52.1707/95).

EMBRAPA Algodão, Núcleo de Goiás, Caixa Postal 714, 74.001-970, Goiânia, GO.

${ }^{3}$ Fundação Universidade do Tocantins-UNITINS, Campus Universitário de Gurupi, Caixa Postal 66, 77.400-000, Gurupi, TO.

${ }^{4}$ EMBRAPA Milho e Sorgo, Caixa Postal 151, 35.701-970, Sete Lagoas, MG. 


\section{INTRODUÇÃO}

No Estado do Tocantins, o cultivo do milho em condições de "terras altas sob cerrado", constitui-se numa das principais atividades agrícolas, perfazendo na safra 1999/ 2000 uma área cultivada próxima a 60 mil ha (Tocantins, 2000). Sendo uma atividade que abrange diversos segmentos de produtores e condições ambientais, os níveis de produtividade são variáveis, cuja a média não ultrapassa $2 \mathrm{t} / \mathrm{ha}$ (Tocantins, 2000). Em cultivos de baixa produtividade, entre as principais limitações, destacam-se os fatores de solo. Neste sentido, a condição de "terras altas", onde situam-se os municípios de Pedro Afonso e Gurupi, apresenta solos ácidos, além de baixos teores de $\mathrm{P}, \mathrm{K}$, $\mathrm{Ca}$ e Mg e matéria orgânica.

A acidez, associada a baixa fertilidade, provoca deficiências nutricionais e restrição no desenvolvimento do sistema radicular, decrescendo a tolerância à seca e a absorção de nutrientes (Olmos \& Camargo, 1975; Foy et al., 1978; Ritchie, 1989). A aplicação de corretivos, práticas rotacionas com culturas de adubação verde e o emprego de material genético adaptado figuram entre as pincipais estratégias para manejo desses solos.

No melhoramento genético visando o desenvolvimento de cultivares adaptados, a população a ser submetida a seleção deve ser portadora de genótipos de constituição alélica superior, com suficiente variabilidade genética. Neste sentido, populações tais como CMS 30 (Composto Amplo),
CMS 36 (Sintético Cerrado), CMS 04 (Amarillo Dentado), CMS 13 (Composto Cerrado) e CMS 14C (Pool 25), pela Embrapa Milho e Sorgo; populações SA-3, SA-4, SA-5, SA-6, SA-7 e SA-8, pelo Centro Internacional de Melhoramento de Milho e Trigo (CIMMYT) e a população TAIÚBA e TAITINGA pelo Instituto Agronômico (IAC), têm sido mencionadas por seus desempenhos promissores e variabilidade genética em condição de solos ácidos e de baixa fertilidade (Bahia Filho et al., 1976; Eleutério et al., 1988; Lima et al., 1988; Paterniani, 1990; Pandey \& Gardner, 1992; Borrero et al., 1995; Santos et al., 1998).

Tendo-se como proposta o melhoramento genético de milho voltado para a adaptação a solos ácidos e de baixa fertilidade em "terras altas sob cerrado", condição representada pelos municípios de Pedro Afonso e Gurupi, e frente a grande diversidade genética na espécie Zea mays L., o presente estudo objetivou identificar preliminarmente populações de milho com performances produtivas superiores, que passarão a integrar estudos de estimação de parâmetros genéticos e, posteriormente, programas de seleção.

\section{MATERIAL E MÉTODOS}

Foram avaliadas um total de 160 populações de milho, com origem do Banco Ativo de Germoplasma da Embrapa Milho e Sorgo, separadas em ensaios de ciclo tardio (76 populações) e ensaios de ciclo precoce (84 populações), em experimentos nos municípios de Pedro Afonso e Gurupi. 
Em Pedro Afonso, ao Centro do Estado ( $8^{\circ} 58 \varnothing$ Sul e $48^{\circ} 11 \varnothing$ Oeste), com altitude de $187 \mathrm{~m}$, a experimental corresponde a um Latossolo Vermelho, com textura média. Nesse local, realizaram-se avaliações nas safras 1994/95, 1995/96 e 1998/99. Em Gurupi, ao Sul do Estado (11 ${ }^{\circ} 43^{\prime}$ Sul e $49^{\circ} 15^{\prime}$ Oeste), com altitude de $230 \mathrm{~m}$, a área experimental corresponde a um Latossolo Vermelho Amarelo, com textura arenosa. Nesse local realizaram-se avaliações nas safras 1994/95 e 1998/ 99. As composições químicas dos solos dessas áreas experimentais encontram-se na Tabela 1. A precipitação média anual na Região Centro-Sul do Estado é em torno de $1500 \mathrm{~mm}$, com distribuição concentrada nos meses de novembro a março. Nos meses de outubro a março as temperaturas médias diurnas e noturnas variam em torno de $27^{\circ} \mathrm{C}$ e $17^{\circ} \mathrm{C}$, respectivamente (Universidade do Tocantins, 1992).

Na safra 1994/95, os ensaios de ciclo tardio e precoce foram constituídos de 45 populações e quatro testemunhas (BR 201, ZENECA 8452, PIONEER 3069 e AG 510), respectivamente. Os experimentos fo- ram conduzidos de acordo com o delineamento experimental em látice $7 \times 7$, com duas repetições. Na safra 1995/96, 40 populações constituíram o ensaio de ciclo tardio e 48 populações constituíram o ensaio de ciclo precoce. Entre os tratamentos acrescentou-se as mesmas testemunhas empregadas na safra anterior. $\mathrm{O}$ delineamento experimental foi em blocos completos casualizados, com duas repetições. Em 1998/99, 17 populações constituíram o ensaio de ciclo tardio e 16 populações constituíram o ensaio de ciclo precoce. Aos tratamentos foram acrescentadas duas testemunhas (BR 201, ZENECA 8452) entre as quatro empregadas nas avaliações anteriores. $\mathrm{O}$ delineamento experimental foi em blocos completos casualizados, com quatro repetições.

Em todos os experimentos as parcelas experimentais foram de duas fileiras com quatro metros lineares, espaçadas de $1,0 \mathrm{~m}$ e $0,9 \mathrm{~m}$, nos experimentos de ciclo tardio e de ciclo precoce, respectivamente, buscando-se um estande ideal de 40 plantas por parcela. Foram coletados dados referentes as variáveis: número de dias para $50 \%$ de florescimento feminino (FF); altura da planta (AP) e

Tabela 1. Composição química do solo das áreas experimentais de Gurupi e Pedro Afonso .

\begin{tabular}{|c|c|c|c|c|c|c|c|c|c|c|c|c|}
\hline \multirow{2}{*}{$\begin{array}{c}\text { Camada } \\
\mathrm{cm}\end{array}$} & \multirow{2}{*}{$\begin{array}{c}\mathrm{pH} \\
\mathrm{CaCl} 2 \\
\end{array}$} & \multirow{2}{*}{$\begin{array}{l}\text { M.O } \\
\text { g/dm }{ }^{3}\end{array}$} & \multirow{2}{*}{$\begin{array}{c}\mathrm{P} \\
\mathrm{mg} / \mathrm{dm}^{3}\end{array}$} & K & $\mathrm{Ca}$ & $\mathrm{Mg}$ & Al & $\mathrm{H}+\mathrm{Al}$ & SB & $T$ & V & $\mathrm{m}$ \\
\hline & & & & \multicolumn{7}{|c|}{$\mathrm{m} \mathrm{mol} / \mathrm{dm}^{3}$} & $\%$ & $\%$ \\
\hline \multicolumn{13}{|c|}{ GURUPI } \\
\hline $0-20$ & 4,6 & 26 & 2 & 0,5 & 7 & 4 & 2 & 34 & 12 & 46 & 25 & 16 \\
\hline $20-40$ & 4,6 & 19 & 1 & 0,5 & 4 & 2 & 2 & 31 & 7 & 38 & 17 & 28 \\
\hline \multicolumn{13}{|c|}{ PEDRO AFONSO } \\
\hline $0-20$ & 4,8 & 36 & 4 & 1,4 & 17 & 14 & 1,2 & 56 & 32 & 88 & 36 & 4 \\
\hline $20-40$ & 4,6 & 28 & 2 & 1,3 & 12 & 10 & 2 & 54 & 23 & 77 & 30 & 8 \\
\hline
\end{tabular}


altura da espiga (AE), em cm; e peso de grãos (PG), em kg/parcela. Os dados de peso de grãos foram corrigidos para $o$ estande ideal, conforme apresentado em Vencovsky \& Barriga (1992). Para variável PG corrigidos para estande ideal, considerando-se fixo os efeitos de tratamentos e de locais, realizaram-se análises da variância ao nível de locais e conjuntas de locais, nas safras em que houveram experimentos e Pedro Afonso e Gurupi. Para possibilitar a comparação entre médias de PG, foram obtidas as diferenças mínimas significativas pelo teste de Tukey. São apresentadas as médias de FF, AP e AE e PG para as populações com as maiores médias de produtividade $(30 \%$ superiores) em cada experimento.

\section{RESULTADOS E DISCUSSÃO}

Nas avaliações na safra 1994/95, em ensaios tardio e precoce e em ambos os locais, verificou-se comportamentos produtivos diferenciados entre populações $(\mathrm{P}<0,01)$. Em análise conjunta de locais, a interação populações $\mathrm{x}$ locais também foi significativa $(\mathrm{P}<0,05)$ em ambos os ensaios, indicando que os locais exerceram efeitos diferenciados sobre a performance produtiva das populações.

Tendo-se definido a priori o efeito de locais como fixo, bem como devido a ocorrência da interação populações $\mathrm{x}$ locais, a identificação das populações de melhor performance deve ser ao nível de cada local, explorando-se positivamente a interação. Neste sentido, nas avaliações em Pedro Afonso na safra 1994/95, entre as populações melhor desempenho produtivo (30\% superiores), destacaram- se as populações tardias IAPAR 51, EGO 17 e CMS 08, com produtividades superiores a $7 \mathrm{t} / \mathrm{ha}$, próximo ao desempenho da melhor testemunha BR 201. Essas populações foram em torno de $2 \mathrm{t} / \mathrm{ha}$ mais produtivas que a população CMS 50, de melhor desempenho no ensaio precoce (Tabela 2). Para os caracteres FF, $\mathrm{APe} \mathrm{AE}$, essas populações obtiveram valores de 63 a 64 dias, 212 a $217 \mathrm{~cm}$ e 101 a $116 \mathrm{~cm}$, respectivamente (Tabela 2), superiores aos verificados nas testemunhas, porém, próximos a valores verificados em cultivares avaliados nesses locais (Morello et al., 1997; Peluzio et al., 1998; Leão et al., 1999).

Em Gurupi, ainda na safra 1994/ 95, as melhores performances produtivas foram obtidas pelas popualações K 9201 e CMS 39, com produtividades superiores a $5 \mathrm{t} / \mathrm{ha}$, também pertencentes ao ensaio tardio (Tab. 2). Esses desempenhos foram superiores aos obtidos pelas testemunhas ZENECA 8452 e BR 201. Em comparação a população EGO 503, de maior produtividade no ensaio precoce, essas populações foram em torno de 1,4 t/ha mais produtivas. Para FF, AP e AE, as médias destas populações foram de 51 e 57 dias, 160 e $189 \mathrm{~cm}$ e 80 e $96 \mathrm{~cm}$, respectivamente. Esses valores são inferiores aos verificados em Pedro Afonso, porém coerentes com o maior nível de estresse na condição de cultivo de Gurupi.

Em média, a produtividade das populações tardias em Pedro Afonso superou em $1 \mathrm{t} / \mathrm{ha}$ a produtividade deste mesmo grupo de populações em Gurupi, comportamento também 
verificado no ensaio precoce, com produtividade de $0,7 \mathrm{t} / \mathrm{ha}$ superior em Pedro Afonso. Estes resultados evidenciam as diferenças previamente conhecidas entre os dois locais, ressaltando-se a melhor condição de Pedro Afonso, principalmente em relação a fatores de solo (Tab. 1).

Nas avaliações em 1995/96, apenas em Pedro Afonso, os comportamentos produtivos entre as populações foram distintos $(\mathrm{P}<0,01)$, tanto no experimento tardio quanto no experimento precoce. As populações COMPOSTO TERRAS BAIXAS E TAIÚBA, pertencentes ao ensaio tardio, apresentaram as maiores produtividades, em torno de 5,9 t/ha, superando as testemunhas PIONEER 3069, ZENECA 8452, BR 201 e AG

Tabela 2. Médias das populações de maior produtividade ( $30 \%$ superiores) em Pedro Afonso e Gurupi, em ensaios de ciclo tardio e precoce, na safra 1994/95.

\begin{tabular}{|c|c|c|c|c|c|c|c|c|c|}
\hline \multicolumn{10}{|c|}{ Pedro Afonso } \\
\hline \multicolumn{5}{|c|}{ Tardio } & \multicolumn{5}{|c|}{ Precoce } \\
\hline Populações & $\mathrm{FF}$ & AP & $\mathrm{AE}$ & $P G$ & Populações & $\mathrm{FF}$ & AP & $\mathrm{AE}$ & $P G$ \\
\hline IAPAR 51 & 63 & 215 & 101 & 7,46 & CMS 50 & 61 & 216 & 107 & 5,02 \\
\hline EGO 17 & 63 & 212 & 107 & 7,11 & EGO 19 & 57 & 215 & 114 & 4,96 \\
\hline CMS 08 & 64 & 217 & 116 & 7,06 & PE 001 & 56 & 193 & 111 & 4,69 \\
\hline EGO 18 & 64 & 215 & 97 & 6,53 & V $8321-18$ & 56 & 170 & 79 & 4,32 \\
\hline PMI 8505 & 63 & 209 & 114 & 6,40 & J 9201 & 54 & 192 & 86 & 4,31 \\
\hline BR 105 & 66 & 212 & 102 & 5,60 & F 8336 & 56 & 185 & 96 & 4,27 \\
\hline CMS 07 & 61 & 187 & 82 & 5,52 & CMS 53 & 52 & 178 & 75 & 4,23 \\
\hline POOL 22 & 60 & 209 & 105 & 5,30 & BR 451 & 58 & 186 & 79 & 4,19 \\
\hline IAPAR 50 & 65 & 182 & 79 & 5,15 & K 9202 & 59 & 201 & 106 & 4,17 \\
\hline C. CANAFORTE' & 56 & 209 & 108 & 5,04 & BR 106 & 59 & 206 & 105 & 4,16 \\
\hline CMS 39 & 64 & 201 & 85 & 4,92 & EGO 503 & 51 & 181 & 89 & 4,08 \\
\hline IAPAR 26 & 60 & 174 & 81 & 4,87 & F 7928 & 50 & 188 & 92 & 4,06 \\
\hline CMS $14 \mathrm{C}$ & 65 & 209 & 116 & 4,80 & K 9201 & 59 & 216 & 110 & 4,05 \\
\hline IAPAR 50 & 67 & 197 & 105 & 4,78 & TEEY POP & 56 & 153 & 61 & 4,04 \\
\hline TAIÚBA & 64 & 187 & 90 & 4,73 & CMS 22 & 51 & 181 & 94 & 3,99 \\
\hline $\mathrm{BR} 201^{\mathrm{T}}$ & 60 & 194 & 97 & 7,39 & AG $510^{\top}$ & 54 & 200 & 104 & 5,98 \\
\hline ZENECA $8452^{\top}$ & 62 & 190 & 99 & 5,00 & ZENECA $8452^{\top}$ & 50 & 169 & 95 & 5,87 \\
\hline AG $510^{\top}$ & 61 & 179 & 83 & 3,90 & PIONEER $3069^{\top}$ & 53 & 185 & 82 & 4,99 \\
\hline PIONEER $3069^{\top}$ & 59 & 177 & 94 & 3,65 & BR $201^{\top}$ & 53 & 187 & 92 & 3,61 \\
\hline Média Geral & 62 & 193 & 94 & 4,53 & Média Geral & 54 & 182 & 87 & 3,82 \\
\hline D.M.S Tukey ${ }_{(0.05)}$ & & & & 1,40 & D.M.S Tukey $_{(0.05)}$ & & & & 1,78 \\
\hline
\end{tabular}




\begin{tabular}{|c|c|c|c|c|c|c|c|c|c|}
\hline \multicolumn{10}{|c|}{ Gurupi } \\
\hline \multicolumn{5}{|c|}{ Tardio } & \multicolumn{5}{|c|}{ Precoce } \\
\hline Populações & $\mathrm{FF}$ & AP & $\mathrm{AE}$ & $P G$ & Populações & $\mathrm{FF}$ & AP & $\mathrm{AE}$ & $P G$ \\
\hline K 9201 & 63 & 160 & 80 & 5,63 & EGO 503 & 52 & 142 & 64 & 4,25 \\
\hline CMS 39 & 59 & 189 & 96 & 5,42 & $F 8336$ & 53 & 139 & 62 & 3,98 \\
\hline EGO 17 & 52 & 164 & 83 & 4,69 & CMS 37 & 56 & 152 & 70 & 3,71 \\
\hline EGO 18 & 62 & 164 & 80 & 4,58 & J 9201 & 52 & 177 & 85 & 3,69 \\
\hline CMS 36 & 59 & 169 & 80 & 4,58 & F 7928 & 53 & 156 & 66 & 3,60 \\
\hline CMS 03 & 61 & 166 & 76 & 4,53 & V $8321-18$ & 50 & 146 & 63 & 3,59 \\
\hline IAPAR 51 & 60 & 160 & 72 & 4,21 & EGO 15 & 52 & 163 & 79 & 3,59 \\
\hline PMI 8505 & 62 & 177 & 70 & 4,16 & EGO 19 & 50 & 181 & 86 & 3,59 \\
\hline CMS 08 & 63 & 178 & 97 & 4,06 & SINTÉTICO 06 & 53 & 129 & 50 & 3,43 \\
\hline CMS 04C & 61 & 152 & 68 & 3,86 & PE 001 & 55 & 155 & 80 & 3,41 \\
\hline POOL 22 & 58 & 182 & 94 & 3,77 & K 9201 & 56 & 166 & 78 & 3,41 \\
\hline CMS 28 & 62 & 182 & 81 & 3,72 & ACROSS 8528 & 50 & 153 & 59 & 3,39 \\
\hline BR 105 & 63 & 185 & 80 & 3,59 & BR 106 & 50 & 151 & 72 & 3,27 \\
\hline TAIÚBA & 53 & 207 & 110 & 3,53 & C. CANAFORTE ${ }^{2}$ & 46 & 149 & 57 & 3,25 \\
\hline BR 112 & 62 & 182 & 83 & 3,50 & BR 451 & 52 & 148 & 63 & 3,12 \\
\hline AG $510^{\top}$ & 60 & 182 & 89 & 6,07 & BR $201 T$ & 53 & 155 & 69 & 5,74 \\
\hline PIONEER $3069^{\top}$ & 60 & 147 & 66 & 5,43 & ZENECA $8452^{\top}$ & 52 & 145 & 61 & 4,05 \\
\hline ZENECA $8452^{\top}$ & 63 & 155 & 73 & 5,20 & AG $510^{\top}$ & 50 & 180 & 92 & 3,85 \\
\hline$B R 201^{\top}$ & 63 & 142 & 59 & 3,38 & PIONEER $3069^{\top}$ & 55 & 124 & 54 & 2,57 \\
\hline Média Geral & 57 & 162 & 75 & 3,50 & Média Geral & 51 & 143 & 63 & 3,11 \\
\hline D.M.S Tukey ${ }_{(0.05)}$ & & & & 1,99 & D.M.S Tukey $_{(0.05)}$ & & & & 1,52 \\
\hline
\end{tabular}

FF: florescimento feminino (dias); AP: altura da planta (cm); AE: altura da espiga (cm); PG: peso de grãos (t/ha); T: testemunha; ' Composto Canaforte; ${ }^{2}$ Composto Canaforte Precoce.

510 (Tab. 3). Com produtividades acima de 5,5 t/ha, destacaram-se no ensaio precoce as populações MUNENG 8531 e TEEY POP CO, superando as testemunhas BR 201, ZENECA 8452 e AG 510 (Tab. 3). Excetuando-se a população MUNENG $8531 \mathrm{com}$ valor de AP bastante baixo, as demais populações apresentaram valores de FF, AP e AE (Tab. 3) compatíveis com os verificados em cultivares (Morello et al. 1997; Peluzio et al., 1988; Leão et al., 1999).

$\mathrm{Na}$ safra 1998/99, foram observados comportamentos produtivos diferenciados entre 
populações $(\mathrm{P}<0,01)$ em ambos os locais e ensaios. A análise conjunta de locais, revelou a não significância da interação populações $x$ locais $(P>0,05)$. Esses efeitos indicam a ocorrência de performances produtivas diferenciadas entre populações, porém, sem comportamentos diferenciais entre as populações devido as mudanças ambientais.

Tabela 3. Médias das populações de maior produtividade (30\% superiores) em Pedro Afonso, em ensaios de ciclo tardio e precoce, na safra 1995/96.

\begin{tabular}{|c|c|c|c|c|c|c|c|c|c|}
\hline \multicolumn{5}{|c|}{ Tardio } & \multicolumn{5}{|c|}{ Precoce } \\
\hline Populações & $\mathrm{FF}$ & AP & $A E$ & $P G$ & Populações & $\mathrm{FF}$ & AP & $\mathrm{AE}$ & $P G$ \\
\hline C.T. BAIXAS1 & 56 & 191 & 103 & 5,92 & MUNENG 8531 & 56 & 149 & 71 & 5,62 \\
\hline TAIÚBA & 55 & 197 & 105 & 5,84 & TEEY POP CO & 52 & 177 & 80 & 5,55 \\
\hline CMS 34 & 57 & 188 & 99 & 5,10 & EGO 503 & 55 & 159 & 77 & 5,13 \\
\hline CMS 03 & 55 & 203 & 115 & 5,06 & POOL 16 & 58 & 180 & 95 & 5,08 \\
\hline CMS 39 & 57 & 164 & 84 & 4,93 & EGO 15 & 51 & 173 & 78 & 5,06 \\
\hline IRAT 81 & 57 & 190 & 108 & 4,89 & CMS 33 & 48 & 166 & 85 & 4,94 \\
\hline BR 111 & 53 & 199 & 100 & 4,87 & INIAP 527 & 60 & 181 & 80 & 4,77 \\
\hline M 28T & 55 & 184 & 105 & 4,84 & CIMCALI SA $190 \mathrm{~B}$ & 54 & 168 & 85 & 4,72 \\
\hline CMS $14 \mathrm{C}$ & 56 & 195 & 115 & 4,83 & CIMCALI AS 491 & 55 & 155 & 76 & 4,57 \\
\hline BR 105 & 60 & 182 & 88 & 4,82 & H 8705-4 & 58 & 176 & 74 & 4,48 \\
\hline H 8425 & 55 & 196 & 104 & 4,76 & CMS 16 & 53 & 154 & 72 & 4,40 \\
\hline H 8644 & 55 & 167 & 77 & 4,71 & CMS 22 & 56 & 171 & 72 & 4,38 \\
\hline CMS 04C & 58 & 177 & 89 & 4,57 & POOL 18018 & 53 & 171 & 79 & 4,30 \\
\hline H 9021 & 56 & 168 & 63 & 4,53 & ACROSS 8530 & 51 & 157 & 84 & 4,29 \\
\hline CMS 13 & 54 & 166 & 84 & 4,48 & K 9201 & 51 & 171 & 77 & 4,16 \\
\hline PIONEER $3069^{\top}$ & 54 & 182 & 104 & 5,64 & PIONEER $3069^{\top}$ & 48 & 167 & 66 & 5,88 \\
\hline ZENECA $8452^{\top}$ & 57 & 200 & 124 & 4,90 & BR $201^{\top}$ & 48 & 164 & 73 & 4,91 \\
\hline BR $201^{\top}$ & 58 & 208 & 97 & 4,36 & ZENECA $8452^{\top}$ & 56 & 195 & 79 & 4,00 \\
\hline$A G 510^{\top}$ & 60 & 207 & 98 & 2,80 & AG $510^{\top}$ & 52 & 173 & 78 & 3,65 \\
\hline Média Geral & 57 & 184 & 94 & 4,12 & Média Geral & 54 & 169 & 78 & 3,98 \\
\hline D.M.S Tukey ${ }_{(0.05)}$ & & & & 2,22 & D.M.S Tukey ${ }_{(0,05)}$ & & & & 2,47 \\
\hline
\end{tabular}

FF: florescimento feminino (dias); AP: altura da planta (cm); AE: altura da espiga (cm); PG: peso de grãos (t/ha); $T$ : testemunha; ${ }^{1}$ Composto Terras Baixas. 
As populações tardias de melhor desempenho produtivo em Pedro Afonso foram TAITINGA, CMS 15, CMS 28, SIKALQ, BR 106 e BR 105, com produtividades superiores a $5,5 \mathrm{t} /$ ha, próxima a produtividade obtida pela testemunha BR 201 (Tab. 4). Essas populações superaram em mais de 0,5 t/ha a população CMS 59 , a mais produtiva no ensaio precoce (Tab. 4). Para os caracteres FF, AP e AE os valores foram coerentes com os verificados em cultivares (Morello et al. 1997; Peluzio et al., 1988; Leão et al., 1999), não sendo restritivos ao emprego destas populações. As populações de melhor performance em Gurupi, também na safra $1998 / 99$, foram as populações BR 105 e CMS 03, com produtividades acima de $5 \mathrm{t} / \mathrm{ha}$, pertencentes ao ensaio tardio, superando o desempenho da testemunha BR 201 (Tab. 4). Entre as populações do ensaio precoce, a maior produtividade foi obtida pela população CMS 50, em torno de 4,2 t/ha (Tab. 4).

A adaptação diferencial de germoplasma a solos ácidos e com restrição nutricional tem sido mencionada em diversos estudos (Bahia Filho et al., 1976; Naspolini Filho et al., 1981; Eleutério et al., 1988; Lima et al. 1992; Santos et al. 1994; Santos et al., 1998), havendo referências concordantes quanto as populações BR 105 (Suwan DMR), CMS 39 (Composto Nacional), TAIÚBA, CMS 03 (Amarillo Cristalino), TAITINGA e SIKALQ, entre as que se destacaram neste estudo. Ressalta-se que a produtividade de algumas populações em nível próximo as testemunhas, como os híbridos BR 201 e ZENECA 8452, com bons desempenhos em avaliações em Pedro Afonso e Gurupi (Morello et al., 1996; Morello et al., 1997), também é um indicativo da constituição genética favorável a manifestação do potencial produtivo e de outros caracteres agronômicos relevantes, alertando para as possibilidades de emprego em programas de seleção recorrente.

A predominância das populações tardias entre as de melhor performance pode estar relacionada ao maior período de atividade fotossintética e acúmulo de fotossintetatos nestas populações, proporcionando melhores desempenhos produtivos. Estas populações também apresentaram os maiores valores de AP e $\mathrm{AE}$, o que também é esperado, haja vista a elevada correlação genética entre estes caracteres com a produtividade (Hallauer \& Miranda Filho, 1995).

Tendo-se como objetivo principal identificar germoplasma com adaptação à condição de "terras altas sob cerrado" no Estado do Tocantins, os resultados deste estudo alertam para as populações IAPAR 51, EGO 17, CMS 08, CMS 50, COMPOSTO TERRAS BAIXAS, TAIÚBA, TAITINGA, CMS 28, CMS 15 e SIKALQ, em Pedro Afonso; e K9201, CMS 39, BR 105 e CMS 03, em Gurupi; como promissoras, sendo candidatas a estudos de capacidade combinatória e padrões heteróticos, definindo-se as possibilidades de melhoramento.

\section{CONCLUSÕES}

Verificou-se uma expressiva diversidade genética entre as populações e portanto diferentes níveis 
de adaptação às condições de "terras altas sob cerrado" nos municípios de Pedro Afonso e Gurupi, estado do Tocantins".

Os municípios de Pedro Afonso e Gurupi, embora pertencentes a condição de "terras altas sob cerrado", apresentaram diferenças expressivas em PG, FF, AP e AE, justificando terse definido a priori os efeitos fixos de locais. Os efeitos significativos das interações populações $\mathrm{x}$ locais também alertam para a necessidade da condução de programas específicos de

Tabela 4. Médias das populações de maior produtividade (30\% superiores) em Pedro Afonso e Gurupi, em ensaios de ciclo tardio e precoce, na safra 1998/99.

\begin{tabular}{|c|c|c|c|c|c|c|c|c|c|}
\hline \multicolumn{10}{|c|}{ Pedro Afonso } \\
\hline \multicolumn{5}{|c|}{ Tardio } & \multicolumn{5}{|c|}{ Precoce } \\
\hline Populações & FF & AP & $A E$ & $P G$ & Populaçőes & $\mathrm{FF}$ & AP & $A E$ & $P G$ \\
\hline TAITINGA & 63 & 229 & 128 & 5,72 & CMS 59 & 52 & 216 & 107 & 5,02 \\
\hline CMS 15 & 59 & 220 & 122 & 5,68 & CMS 53 & 53 & 215 & 114 & 4,96 \\
\hline CMS 28 & 52 & 216 & 120 & 5,65 & CMS 50 & 56 & 193 & 111 & 4,69 \\
\hline SIKALQ & 62 & 235 & 138 & 5,58 & Sint. Dent ${ }^{1}$ & 52 & 170 & 79 & 4,32 \\
\hline BR 106 & 59 & 211 & 129 & 5,58 & PC 9501 & 53 & 192 & 86 & 4,31 \\
\hline BR 105 & 61 & 245 & 116 & 5,55 & CMS 51 & 50 & 185 & 96 & 4,27 \\
\hline ZENECA $8452^{\top}$ & 59 & 178 & 91 & 6,90 & ZENECA $8452^{\top}$ & 56 & 190 & 100 & 6,15 \\
\hline BR 201 & 57 & 87 & 180 & 5,67 & BR 201 & 56 & 182 & 96 & 4,85 \\
\hline Média Geral & 63 & 231 & 126 & 5,23 & Média Geral & 52 & 220 & 122 & 5,21 \\
\hline D.M.S Tukey ${ }_{(0.05)}$ & & & & 0,83 & D.M.S Tukey ${ }_{\{0.05\}}$ & & & & 1,10 \\
\hline \multicolumn{10}{|c|}{ Gurupi } \\
\hline \multicolumn{5}{|c|}{ Tardio } & \multicolumn{5}{|c|}{ Precoce } \\
\hline Populações & FF & AP & $\mathrm{AE}$ & $P G$ & Populaçōes & $\mathrm{FF}$ & AP & $\mathrm{AE}$ & $P G$ \\
\hline BR 105 & 61 & 206 & 116 & 5,35 & CMS 50 & 52 & 142 & 64 & 4,25 \\
\hline CMS 03 & 63 & 200 & 119 & 5,13 & CMS 59 & 52 & 139 & 62 & 3,98 \\
\hline TAIỦBA & 61 & 184 & 105 & 4,96 & Sint. Dent1 & 52 & 152 & 70 & 3,71 \\
\hline CUPURICO DMR & 61 & 175 & 101 & 4,86 & PC 9502 & 51 & 177 & 85 & 3,69 \\
\hline IAPAR 51 & 64 & 185 & 94 & 4,84 & CMS 57 & 59 & 156 & 66 & 3,60 \\
\hline CMS $04 \mathrm{C}$ & 65 & 199 & 110 & 4,72 & CMS 23 & 52 & 146 & 63 & 3,59 \\
\hline ZENECA $8452^{\top}$ & 54 & 170 & 85 & 6,12 & ZENECA $8452^{\top}$ & 59 & 180 & 90 & 6,91 \\
\hline $\mathrm{BR} 201^{\top}$ & 56 & 175 & 83 & 4,72 & BR $201^{\top}$ & 58 & 171 & 87 & 5,32 \\
\hline Média Geral & 60 & 190 & 106 & 4,66 & Média Geral & 51 & 192 & 100 & 5,73 \\
\hline D.M.S Tukey ${ }_{(0.05)}$ & & & & 0,98 & D.M.S Tukey ${ }_{(0.05)}$ & & & & 0,99 \\
\hline
\end{tabular}

FF: florescimento feminino (dias); AP: altura da planta (cm); AE: altura da espiga (cm); PG: peso de grãos (t/ha); $\mathrm{T}$ : testemunha; ${ }^{1}$ Sintético Dentado. 
melhoramento nestes locais.

As populações de melhor performance produtiva e com comportamentos agronômicos satisfatórios em termos de $\mathrm{FF}, \mathrm{AP}$ e $\mathrm{AE}$ poderão ser utilizadas em futuros programas de melhoramento genético intrapopulacional e/ou interpopulacional.

\section{Bibliografia citada}

Bahia Filho, A.F.C.; França, G.E.; Pitta, G.V.E.; Magnavaca, R.; Mendes, J.F; Bahia, F.G.F. T.C.; Pereira, P. 1976. Avaliação de linhagens e populações de milho em condições de elevada acidez. In: Reunião Brasileira de Milho e Sorgo, 11., 1976, Piracicaba. Anais... ESALQ, Piracicaba. p.51-58.

Borrero, J.C.; Pandey, S.; Ceballos, H.; Magnavaca, R.; Bahia Filho, A.F.C. 1995. Genetic variances for tolerance to soil acidity in a tropical maize population. Maydica, 40(3):283-288.

Eleutério, A.; Gama, E.E.G.; Morais, A.R. 1988. Capacidade de combinação e heterose em híbridos intervarietais de milho adaptados às condições de cerrado. Pesquisa Agropecuária Brasileira, 23(1):247-253.

Foy, C.D.; Chaney, R.L.; White, M.C. 1978. The physiology of metal toxicity in plants. Annual Review of Plant Physiology, 29:511-566.

Hallauer, A.; Miranda Filho, J.B. 1995. Quantitative genetics in maize breeding. Iowa State University Press, Ames. 468p.

Leão, F.F.; Peluzio, J.M.; Silva, R.R.; Reis, M.S.; Santos, M.G. 1999. Avaliação de cultivares de milho no Estado do Tocantins-safra 1998/99. Comunicado Técnico n. 11. Fundação Universidade do Tocantins/Faculdade de Engenharia Agronômica, Palmas. 20p.

Lima, M.; Furlani, P.R.; Miranda Filho, J.B. 1992. Divergent selection for aluminum tolerance in a maize (Zea mays L.) popu- lation. Maydica, 37(1):123-132.

Lima, M.; Vitti, P.; Gallo, P.B. 1988. Populações de milho: Características agronômicas e tecnológicas. Bragantia, 47(2):55-62.

Morello, C.L.; Coêlho, R.M.S.; Fernandes, D.M.; Peluzio, J.M.; Sá Filho, J.D.; Gomes, I.R.; Coimbra, R.R. 1996. Avaliação de cultivares de milho no Estado do Tocantins-Safra 1995/96. Comunicado Técnico n. 2. Universidade do Tocantins/Faculdade de Engenharia Agronômica, Gurupi. 14p.

Morello, C.L.; Fernandes, D.M.; Peluzio, J.M.; Coimbra, R.R. 1997. Avaliação de cultivares de milho no Estado do Tocantins-safra 1996/97. Comunicado Técnico n. 3. Universidade do Tocantins/ Faculdade de Engenharia Agronômica, Gurupi. 22p.

Naspolini Filho, V.; Bahia Filho, A.F.C.; Vianna, R.T.; Gama, E.E.G.; Vasconcelos, C.A.; Magnavaca, R. 1981. Comportamento de linhagens e de híbridos simples de milho (Zea mays $\mathrm{L}$.) em solos sob vegetação de cerrado. Ciência e Cultura, 33(5):722-727.

Olmos, J.I.L.; Camargo, M.N. 1975. Ocorrência de alumínio tóxico nos solos do Brasil, sua caracterização e distribuição. Ciência e Cultura, 28(2):171-180.

Pandey, S.; Gardner, C.O. 1992. Recurrent selection for population, variety, and hybrid improvement in tropical maize. Advances in Agronomy, 48:1-87.

Paterniani, E. 1990. Maize breeding in the tropics. Critical Reviews in Plant Sciences, 9:125-154.

Peluzio, J.M.; Leão, F.F.; Morello, C.L.; Santos, G.R.; Didonet, J. 1988. Avaliação de cultivares de milho no Estado do Tocantins-safra 1997/98. Comunicado Técnico n. 4. Universidade do Tocantins/ Faculdade de Engenharia Agronômica, Palmas. 16p.

Ritchie, G.S.P. 1989. The chemical behaviour of aluminum, hydrogen and manganese in 
acid soils. In: ROBSON, A.D. Soil acidity and plant growth. Academic Press Australia, Marrickville. 306p.

Santos, MX; Carvalho, H.W.L.; Leite, C.E.P.; Andrade, R.V.; Vasconcelos, C.A. 1998. Evaluation and selection of tropical maize (Zea Mays L.) accessions in low-fertility soils with phosphorus limitations. Plant Genetic Resources Newsletter, 113:17-21.

Santos, MX.; Pacheco, C.A.P.; Guimarães, P.E.O,; Gama, E.E.G.; Silva, A.E.; Oliveira, A.C. 1994. Diallel among twenty eight varieties of maize. Brazilian Journal of Genetics, 17(3):277-282.

Tocantins. 2000. Informativo conjuntural. Secretaria de Estado da Produção, Palmas. 5p.

Universidade do Tocantins. 1992. I plano diretor de pesquisa agropecuária e florestal do Estado do Tocantins. Universidade do Tocantins, Gurupi. 110p.

Vencovsky, R.; Barriga, P. 1992. Genética biométrica no fitomelhoramento. Sociedade Brasileira de Genética, Ribeirão Preto. 496p. 